\title{
The Molecular Interactions between Filtered Proteins and Proximal Tubular Cells in Proteinuria
}

\author{
Richard J. Baines Nigel J. Brunskill \\ Department of Infection, Immunity and Inflammation, University of Leicester School of Medicine, Leicester, UK
}

\section{Key Words}

Endocytosis · Nephropathy · Proteinuria • Proximal tubule •

Signal transduction

\begin{abstract}
Proteinuria is associated with progressive chronic kidney disease and poor cardiovascular outcomes. Exposure of proximal tubular epithelial cells to excess proteins leads to the development of proteinuric nephropathy with tubular atrophy, interstitial inflammation and scarring. Numerous signalling pathways are activated in proximal tubular epithelial cells under proteinuric conditions resulting in gene transcription, altered growth and the secretion of inflammatory and profibrotic mediators. Megalin, the proximal tubular scavenger receptor for filtered macromolecules, has intrinsic signalling functions and may also link albumin to growth factor receptor signalling via regulated intramembrane proteolysis. It now seems that endocytosis is not always a prerequisite for albumin-evoked alterations in proximal tubular cell phenotype. Recent evidence shows the presence of other potential receptors for proteins, such as the neonatal Fc receptor and CD36, in the proximal tubular epithelium.
\end{abstract}

Copyright $\odot 2008$ S. Karger AG, Basel
In renal diseases, proteinuria correlates closely with the rate of progressive chronic kidney disease. Renal function loss under these circumstances is accompanied by the development of a common pattern with tubular atrophy, interstitial inflammation and fibrosis culminating in the end-stage kidney. Many studies have demonstrated a positive correlation between the degree of proteinuria and disease progression, and confirmed the significant renoprotection afforded by antiproteinuric strategies. In the last 15 years, the importance of proteinuria as a marker and maker of kidney disease has been extensively examined.

\section{Activation and Altered Proximal Tubule Epithelial Cell Phenotype in Proteinuria}

The clinicopathological associations described above led to the hypothesis that bioactive macromolecules abnormally filtered into the proteinuric proximal tubule (PT) may be toxic, causing dysregulated signalling and disturbed phenotype in PT epithelial cells (PTEC).

This hypothesis has been extensively tested using in vitro approaches with cultured PTEC, and in vivo with a variety of animal models. The bulk of these studies have focussed on PTEC exposure to albumin, the most prevalent protein in proteinuric glomerular filtrate, but other

Department of Infection, Immunity and Inflammation, Medical Sciences Building

University of Leicester School of Medicine, University Road Leicester LE1 9HN (UK)

Tel. +44 116258 8043, Fax +44 116258 4764, E-Mail njb18@le.ac.uk 
Table 1. Alterations in PTEC signalling, transcription factor activity and secretory phenotype demonstrated under proteinuric conditions in vitro and in vivo (see Pollock and Poronnik [1] and Geckle [2] and the further references therein)

\begin{tabular}{|c|c|c|}
\hline Signalling mediators stimulated & Transcription factors altered & Mediators released \\
\hline $\begin{array}{l}\text { ERK } 0.5 \\
\text { MEK1 } \\
\text { p38 MAP kinase } \\
\text { c-jun N-terminal kinase } \\
\text { Ribosomal p } 70^{\text {S6 }} \text { kinase } \\
\text { PKC } \\
\text { PKB } \\
\text { Phosphatidylinositide 3-kinase } \\
\text { NAD(P)H oxidase } \\
\text { Intracellular }\left[\mathrm{Ca}^{2+}\right] \text { (increased) }\end{array}$ & $\begin{array}{l}\text { NFкB } \\
\text { AP-1 } \\
\text { Elk-1 } \\
\text { STAT } \\
\text { HIF/HRE (reduced) } \\
\text { Peroxisome proliferator-activated } \\
\text { receptor- } \gamma \text { (by albumin-bound fatty acids) }\end{array}$ & $\begin{array}{l}\text { Endothelin-1 } \\
\text { MCP-1 } \\
\text { RANTES } \\
\text { Fractalkine } \\
\text { Interleukin- } 8 \\
\text { Osteopontin } \\
\text { Transforming growth factor- } \beta \\
\text { Tumor necrosis factor- } \alpha \\
\text { Connective tissue growth factor } \\
\text { Fibronectin } \\
\text { Collagen } \\
\text { Complement } \\
\text { Reactive oxygen species }\end{array}$ \\
\hline
\end{tabular}

proteins such as immunoglobulin and transferrin have also been tested in this way. The evidence clearly indicates that albumin, albumin-bound molecules and other filtered proteins are able to dynamically regulate PTEC function with activation of multiple signalling enzymes and transcription factors. As a result, numerous changes in cell phenotype and growth characteristics occur generating a proinflammatory environment in the renal tubulointerstitium that favours the development of fibrotic changes characteristic of the failing kidney [1]. A comprehensive list of pathways activated and mediators released by PTEC in proteinuria is provided in table 1. Low concentrations of albumin favour PTEC survival and proliferation, whereas large amounts of albumin and albuminbound molecules such as fatty acids predispose PTEC to apoptosis.

\section{Megalin and Cubilin as Receptors for Albumin Endocytosis in PTEC}

These findings have aroused intense interest in how filtered proteins, especially albumin, are handled in the PT. It has been long recognised that filtered albumin is retrieved from the proximal tubular lumen by endocytosis. Solute removal by non-specific, fluid-phase endocytosis in the PT is trivial. Specific binding sites for albumin have been described in PTEC, and receptors responsible for albumin re-absorption by receptor-mediated endocytosis have been identified.
Megalin is a giant glycoprotein member of the lowdensity lipoprotein receptor (LDL-R) gene family. In the kidney, it is highly expressed in microvilli and coated pits of the PT brush border and in components of the PTEC subluminal endocytic apparatus [2]. In extrarenal tissues, megalin is also expressed in the luminal membranes of other absorptive epithelia. Structurally megalin is composed of a very large extracellular domain composed of four cysteine-rich ligand-binding areas, 17 epidermal growth factor (EGF)-type repeats and 8 spacer regions possessing YWTD repeats. A short transmembrane region links the extracellular domain to a short cytoplasmic tail of 209 amino acids in humans. Cubilin is a 460$\mathrm{kDa}$ giant extracellular protein anchored to the cell surface but lacking either a transmembrane or intracellular domains. In cultured PTEC, efficient receptor-mediated endocytosis of albumin and a variety of other predominantly low-molecular-weight proteins is dependent on normal expression of both megalin and cubilin. In PTEC, megalin functions in tandem with cubilin to form a lowaffinity, high-capacity system for the re-absorption of a number of filtered proteins [2]. In accordance with these observations, megalin-deficient mice, cubilin-deficient dogs, and humans with cubilin mutations in ImerslundGräsbeck syndrome all show up to a 25 -fold increase in albuminuria.

An absorptive complex composed of several membrane and cytoplasmic proteins is required for the uptake of bound albumin into the cell [2]. The NPXY domain of the megalin cytoplasmic tail (meg-CT) interacts with the 
clathrin coat of membrane invaginations at the base of microvilli leading to ligand/receptor internalisation and shuttling of the receptor between the plasma membrane and the endosomal compartment [2]. The binding affinity of megalin for its ligands is $\mathrm{pH}$ dependent and constant around $\mathrm{pH} 7$, equivalent to that in the PT. In the endosomal vesicles, $\mathrm{pH}$ falls due to combined activity of the vacuole-type $\mathrm{H}^{+}$-ATPase, $\mathrm{Na}^{+} / \mathrm{H}^{+}$exchanger-3, and the $\mathrm{ClC}-5$ chloride channel. Megalin ligands dissociate from the receptor at $\mathrm{pH}<6.5$. Ultimately the receptor is recycled to the plasma membrane and its protein ligand transferred to the lysosome where it is degraded and constituent amino acids are re-absorbed into the circulation [2].

\section{Megalin - More than an Endocytic Receptor?}

Conventionally megalin has been regarded as a scavenger receptor, and its ligands such as albumin simply as inert cargo destined for degradation. Several lines of evidence now suggest that megalin has signalling functions beyond its role in endocytosis. The megalin knockout mouse is barely viable, the few pups that survive demonstrate lung and brain abnormalities, proteinuria and a markedly attenuated endocytic apparatus in the PT. The severity of this phenotype is difficult to explain by just the loss of megalin-mediated endocytosis and suggests more diverse functions for megalin. The meg-CT, longer than those of the other LDL-R, possesses sequence motifs potentially involved in signalling. There are multiple consensus sequences for phosphorylation by protein kinases $\mathrm{A}$ and $\mathrm{C}$ (PKC), glycogen synthase kinase-3, and casein kinase II. Meg-CT is phosphorylated on serine by glycogen synthase kinase- 3 which may impact on endocytic function by negatively regulating megalin recycling [3]. Whether other kinases can also phosphorylate meg$\mathrm{CT}$, and how phosphorylation may be agonist regulated are unresolved questions.

Meg-CT also has sequence regions that regulate protein-protein interactions. These include Src homology domains 2 and 3 that mediate interaction with the p85 regulatory subunit of phosphatidylinositide 3-kinase, and proline-rich regions [4]. Identified meg-CT binding partners include the MAP kinase scaffold proteins disabled 1 and 2, JNK interacting proteins 1 and 2, post-synaptic density protein-95-like membrane-associated guanylate cyclase proteins, and other adaptor-type proteins such as ANKRA, SEMCAP-1 and megalin-binding protein [5]. Collectively these associations implicate meg-CT in raf-1 kinase and phosphoinositide signalling, JNK scaffold assembly, G-protein activation and nuclear transcription.

Megalin also anchors protein kinase B (PKB) to the plasma membrane. This kinase is a key component of signal transduction pathways regulating cell survival [4]. In vitro, albumin at the physiological concentrations found in the healthy PT stimulates this megalin-associated $\mathrm{PKB}$, allowing its dissociation from megalin to activate phosphatidylinositide 3-kinase and downstream cell survival pathways. Conversely, higher pathophysiological albumin concentrations downregulate megalin expression in PTEC; consequently, plasma membrane PKB levels are reduced, cell survival pathways are not activated and the cells become sensitised to apoptosis [4]. Therefore, by coupling albumin binding to PKB activity megalin acts as an albumin sensor, under low albumin concentrations promoting cell survival via PKB, but under higher albumin concentrations predisposing to apoptosis. Consistent with this, in proteinuric kidneyspecific megalin knockout mice with a mosaic pattern of PTEC megalin deficiency, the apoptotic rate was significantly greater in megalin-deficient cells [6]. These data may explain why in PTEC low concentrations of albumin may be protective and high concentrations detrimental.

\section{Regulated Intramembrane Proteolysis of Megalin}

Regulated intramembrane proteolysis is a mechanism that links receptor function to transcriptional activity [7]. It is characterised by ectodomain shedding of transmembrane proteins regulated by PKC and performed by the ADAM family of metalloproteases. The remaining membrane-associated C-terminal fragment is then cleaved within the transmembrane region by $\gamma$-secretase and the released C-terminal domain is trafficked to the nucleus where it acts as a transcription factor.

Megalin has been shown to undergo regulated intramembrane proteolysis both constitutively and following interaction with its ligand, the vitamin-D-binding protein [7], although whether all megalin ligands cause shedding is unknown. A role for megalin in the regulation of PTEC gene transcription has recently been described [8]. Overexpression of membrane-bound meg-CT diminishes expression of megalin itself, and $\mathrm{Na}^{+} / \mathrm{H}^{+}$exchanger-3 at both mRNA and protein levels in opossum kidney cells. This effect is attenuated by the inhibition of $\gamma$-secretase [8]. Although meg-CT is predicted to target the nu- 
cleus, this has not yet been demonstrated, probably due to its short half-life in the cell. This ability to regulate PTEC gene expression may further explain how megalin functions as a PT sensor of filtered protein, regulating multiple aspects of PTEC phenotype according to the prevailing luminal microenvironment.

Overall therefore, emerging new evidence suggests that megalin signalling may support homeostatic tubular balance in health when physiological levels of protein are present in the tubular lumen, but if dysregulated in proteinuric disease the result could be PTEC pathology typical of proteinuric nephropathy.

\section{Interaction between LDL-R and Growth Factor Receptors}

Functional inactivation of the EGF receptor (EGF-R) in renal proximal tubular cells reduces tubulointerstitial damage after renal injury. However, EGF itself may not be responsible for this effect. When PTEC are exposed to albumin at high concentrations, comparable to those found under proteinuric PT conditions, expression of EGF-R is increased, EGF-R activity is enhanced and ERK stimulated via an EGF-R-dependent pathway [9]. This effect is inhibited by the specific EGF-R inhibitor AG-1478 which inhibits the intrinsic tyrosine kinase activity of the receptor [9]. Therefore, albumin per se appears able to activate EGF-R. The localisation of the EGF-R within polarised cells will significantly govern its pathophysiological role. In health around $10 \%$ of PTEC EGF- $R$ is localised to the apical membrane, but in diseased kidneys apical EGF-R expression is much increased [10].

The mechanism of albumin-evoked stimulation of EGF-R remains uncertain. Six EGF-like activators of EGF-R have been described: EGF, transforming growth factor- $\alpha$, heparin-binding EGF, amphiregulin, betacellulin and epiregulin. Each of these mediators exists as a transmembrane precursor that must undergo ectodomain cleavage to release the soluble EGF-activating fragment. Thus, the extracellular release of protein fragments with EGF-like sequences results in EGF-R activation [11]. Crucially in this context, the extracellular domain of megalin has 17 EGF-type repeats. It is therefore conceivable that if ectodomain shedding of megalin occurs from PTEC in the pathological setting of proteinuria then abnormal EGF activation could occur with resulting tubulointerstitial injury. Alternatively, transactivation of the EGF-R by G-protein-coupled receptors could in part be regulated by PKC and c-Src, both of which activate ERK and are stimulated by albumin in PTEC. From these studies it is difficult to ascertain whether this dependency lies up- or downstream of the EGF-R. In other cell types there is little indication that albumin is able to transactivate the EGF-R and hence the mechanism may be restricted to the PTEC.

\section{Is Endocytosis a Prerequisite for Albumin Signalling?}

It is generally assumed that albumin 'overload' of PTEC is an obligate requirement before adverse responses are manifest. However, the signalling capacities of megalin discussed above argue that endocytosis may not always be necessary. Furthermore, Diwaker et al. [12] demonstrated that opossum kidney proximal tubular cells, which show avid albumin endocytosis, and HKC-8 proximal tubular cells, which display negligible albumin endocytosis, released an equivalent amount of transforming growth factor- $\beta$ when exposed to albumin. We have recently studied the release of MCP-1 by mouse primary cultures of PTEC. These cells exhibit substantial albumin endocytosis that can be similarly blocked by both statins and thiazolidinediones, but only statins had the ability to block MCP-1 production [13]. Therefore, absent or inhibited endocytosis is insufficient to protect PTEC from adverse effects of albumin exposure.

\section{Are Other Receptors for Proteins Present in PTEC?}

In non-renal tissues, other albumin-binding proteins are described. The neonatal $\mathrm{Fc}$ receptor $(\mathrm{FcRn})$ transfers passive humoral immunity from the mother to the fetus, but also serves to conserve IgG by protecting it from degradation throughout life. In addition to binding IgG, the FcRn also binds albumin, diverting it from degradative pathways and increasing its circulating half-life [14]. In the kidney, FcRn is located in podocytes and PTEC. Recent work has shown that podocyte FcRn internalises and clears IgG from the glomerular basement membrane [15]. The function of FcRn expressed in the PT is undetermined, but clearly this would be well placed to mediate re-absorption of filtered albumin and IgG.

Other scavenger receptors are present in the luminal membrane of PTEC. In particular, altered PTEC expression of CD36 has been noted in several models of 
renal disease [16]. CD36 interacts with a variety of ligands including long-chain fatty acids, modified LDL, and advanced glycation end products. Increased expression of CD36 appears to sensitise PTEC to apoptosis in diabetic nephropathy and may mediate inflammatory and albumin-induced profibrotic responses in PTEC [16]. Albumin is a carrier of long-chain fatty acids in the circulation. In proteinuria, the amounts of albuminbound fatty acid are greatly increased and these are carried with albumin into the PT. Potentially, therefore, filtered albumin may interact with PTEC CD36 by virtue of its fatty acid load, although this remains to be demonstrated.

\section{Conclusion}

The study of albuminuria and proteinuric nephropathy has revealed key information about the mechanisms of chronic kidney disease and illuminated new functions for albumin. Unequivocal signalling events induced by filtered proteins occur in PTEC. Once controversial signalling effects of albumin are now explained and justified by new information about albumin receptors in PTEC. Several interesting new players are emerging and deserve further investigation. Given the importance of proteinuria as both a marker and maker of kidney and cardiovascular disease, it seems likely that proteinuria/receptor interactions will remain a vital area for researching the quest for new pharmacological targets in the fight against some of the most important public health problems.

\section{References}

1 Pollock C, Poronnik P: Albumin transport and processing by the proximal tubule: physiology and pathophysiology. Curr Opin Nephrol Hypertens 2007;16:359-364.

2 Geckle M: Renal tubule albumin transport. Ann Rev Physiol 2005;67:573-594.

-3 Yuseff MI, Farfan P, Bu G, Marzolo MP: A cytoplasmic PPPSP motif determines megalin's phosphorylation and regulates receptor's recycling and surface expression. Traffic 2007;8:1215-1230.

-4 Caruso-Neves C, Pinherio AAS, Cai H, Souza-Menezes J, Guggino WB: PKB and megalin determine the survival or death of renal proximal tubule cells. Proc Natl Acad Sci USA 2006;103:18810-18815.

5 Gotthardt M, Trommsdorff M, Nevitt M, Shelton J, Richardson JA, Stockinger W, Nimpf J, Herz J: Interactions of the low density lipoprotein receptor gene family with cytosolic adaptor and scaffold proteins suggest diverse biological functions in cellular communication and signal transduction. J Biol Chem 2000:275:25616-25624.

-6 Theilig F, Kriz W, Jerichow T, Schrade P, Hähnel B, Willnow T, Le Hir M, Bachmann S: Abrogation of protein uptake through megalin-deficient proximal tubules does not safeguard against tubulointerstitial injury. J Am Soc Nephrol 2007;18:1824-1834.
Biemesderfer D: Regulated intramembrane proteolysis of megalin: linking urinary protein and gene regulation in the proximal tubule? Kidney Int 2005;69:1717-1721.

$\checkmark 8$ Li Y, Cong R, Biemesderfer D: The $\mathrm{COOH}$ terminus of megalin regulates gene expression in opossum kidney proximal tubule cells. Am J Physiol Cell Physiol 2008;295: C529-C537. DOI:10.1152/ajpcell.00037. 2008.

-9 $\overline{\text { Reich }} \mathrm{H}$, Tritchler D, Herzenberg AM, Kassiri Z, Zhou X, Gao W, Scholey JW: Albumin activates ERK via EGF receptor in human renal epithelial cells. J Am Soc Nephrol 2005; 16:1266-1278.

10 Gesualdo L, Di Paolo S, Calabro A, Milani S, Maiorano E, Ranieri E, Pannarale G, Schena FP: Expression of epidermal growth factor and its receptor in normal and diseased human kidney: an immunohistochemical and in situ hybridization study. Kidney Int 1996; 49:656-665.

11 Carpenter G: EGF receptor transactivation mediated by the proteolytic production of EGF-like agonists. Sci STKE 2000;15:1-2.
12 Diwaker R, Pearson AC, Colville-Nash P, Brunskill NJ, Dockrell MEC: The role played by endocytosis in albumin-induced secretion of TGF $\beta-1$ by proximal tubular epithelial cells. Am J Physiol 2007;292:F1464F1470.

13 Chana RS, Sidaway JE, Brunskill NJ: Statins but not thiazolidinediones attenuate albumin-mediated chemokine production by proximal tubular cells independently of endocytosis. Am J Nephrol 2008;28:823-830.

14 Anderson CL, Chaudhury C, Kim J, Bronson CL, Wani MA, Mohanty S: Perspective FcRn transports albumin: relevance to immunology and medicine. Trends Immunol 2006;27:343-348.

15 Akilesh S, Huber TB, Wu H, Wang G, Hartleben B, Kopp JB, Miner JH, Derry C, Roopenian DC, Unanue ER, Shaw AS: Podocytes use FcRn to clear IgG from the glomerular basement membrane. Proc Natl Acad Sci USA 2008;105:967-972.

16 Okamura DM, Lopez-Guisa JM, Koelsch K, Collins K, Eddy AA: Atherogenic scavenger receptor modulation in the tubulointerstitium in response to chronic renal injury. Am J Physiol 2007;293:F575-F585. 